\title{
Conocer costos en atención de salud: Una necesidad imperativa
}

\author{
Jorge Jiménez ${ }^{1}$ y Camilo Cid $^{2}$ \\ 1.Profesor de Salud Pública PUC, ex Ministro de Salud, Master of Public Health (Johns Hopkins University), \\ Departamento de Salud Pública Facultad de Medicina PUC. \\ 2. Doctor en Economía (Universität Duisburg-Essen), Profesor Departamento de Salud Pública Facultad de \\ Medicina PUC
}

\section{Introducción:}

En esta edición de la revista chilena de cardiología se publica un interesante trabajo de investigación operacional económica que compara los costos asociados al tratamiento protocolizado de pacientes hipertensos en un hospital tipo IV del Servicio de Salud de Nuble ${ }^{1}$. Más allá del mérito de los autores al poder aplicar técnicas de contabilidad de costos en un establecimiento de la red pública de salud, es interesante poder comentar algunos elementos que surgen de la información recogida, de los métodos aplicados y de la necesidad de poder financiar adecuadamente los prestadores en el ámbito de los servicios públicos.

La tradicional queja de que los números del ente financiero público, FONASA (Fondo Nacional de Salud) están subestimados en relación a los costos reales enfrenta en esta evaluación una prueba interesante. Mucho de la llamada "deuda hospitalaria", fenómeno recurrente en el sistema público desde hace décadas, es probablemente también parte de este mismo síndrome. La suma final del ejercicio financiero de los establecimientos genera déficits operacionales que llegan a representar hasta un 15 a $20 \%$ de los presupuestos anuales de los hospitales, especialmente aquellos más complejos $^{2}$. No obstante, se debe tener en cuenta que no todo el presupuesto que se dirige a los hospitales opera a través de aranceles y que, a su vez, la deuda no depende sólo de ellos; por ejemplo, la deuda depen- de también del hecho que el presupuesto se reajusta (inflacionariamente) con inflactores que impone el Ministerio de Hacienda que son menores que el IPC general, produciéndose una importante pérdida del poder adquisitivo presupuestario año tras año, sólo por este hecho casi trivial. En cualquier caso, es claro que las actividades en los hospitales públicos son el resultado de la atención de las distintas necesidades de salud de la población, asociada a cada uno de ellos, las que varían dependiendo de las condiciones de riesgo sanitario, incluyendo en ellas a las condiciones socio-demográficas y de pobreza. La hipótesis permite señalar que la existencia de deuda responde a una insuficiencia de recursos resultado de una demanda por atenciones de salud que no es completamente financiada por las transferencias presupuestarias.

Las implicancias políticas de estos resultados financieros deficitarios son materia de juicio negativo sobre la eficiencia del sistema público de salud y generan debates recurrentes sobre la calidad de las prestaciones y del uso inadecuado de los recursos. Ello lleva a conclusiones muchas veces interesadas por parte de quienes pretenden, en abstracción de las limitaciones verdaderas de cada situación, a decir que en otros modelos de gestión, como podría ser uno privatizado total o parcialmente, son más eficientes.

Otros comentaristas pueden decir desde una vertiente más macro, que un país como Chile, con expectativas
Correspondencia:

Dr. Jorge Jiménez

Pontificia Universidad Católica de Chile

Santiago

E-mail: jjimenez@med.puc.cl 
de vida al nacer cercanas a los 78 y 80 años y gastos públicos en salud per cápita del orden de 550 USD anuales promedio, son de una alta eficiencia social comparados con otros países de desarrollo avanzado. Todo depende desde el ángulo que se mire el fenómeno. Es importante acotar, sin embargo, que estos cuocientes ventajosos son producto de un mayor énfasis en lo preventivo, de menor costo y mayor efectividad, que en lo curativo. Los años de vida saludables ganados por el sistema de salud chileno, son por así decirlo, más baratos que aquellos que se obtienen en otros países de mayor gasto agregado, principalmente por una mayor densidad de costos de atención médica.

\section{Costos Reales versus Costos Protocolizados}

Los autores del trabajo en análisis comparan el valor resultante de asignar los aranceles de la Modalidad de Atención Institucional (MAI) del FONASA a la canasta de prestaciones para la hipertensión arterial de manejo ambulatorio, de por sí arbitraria y, por lo tanto, no siempre representativa de lo que ocurre en todos los casos la totalidad de las veces, por ello existe siempre una probabilidad de diferencias entre las normas y protocolos y la valorización de ellos. Además, los costos del arancel suponen promedios nacionales, por lo que siempre se encontrarán diferencias en las comparaciones, que no debiesen llamar a alarma, siempre y cuando, se encuentren dentro de un rango razonable. Pero, aún cuando el arancel de la MAI no es directamente comparable con una canasta GES, los autores anotan un costo real significativamente distinto al costo estimado por FONASA para el caso de la Hipertensión, ellos anotan que a nivel unitario las mayores diferencias se observan en la asignación del valor hora profesional de enfermera y nutricionista y en algunos exámenes de laboratorio como el hematocrito y orina. En el total la mayor preponderancia la tienen los recursos humanos de ahí que esta información es preocupante. No obstante, es explicable que en el método de asignación de valor proporcional a cada paciente controlado, existan estas diferencias en valor de recurso humano ya que el valor unitario puede depender del número de controles que cada profesional realice y en el caso de los exámenes, realizados en baja frecuencia y con métodos no automatizados (por lo tanto menos costosos), se generen tales diferencias. Por otra parte, ellos señalan que los costos estimados a través de las guías clínicas de las GES se acercan a lo calculado cuando las etapas de la enfermedad se encuentran en rangos leves en usuarios de bajo riesgo, pero no coinciden en las etapas de mayor riesgo, en las cuales sobrepasan los valores estimados por Fonasa.

Ciertamente el arancel Fonasa está construido sobre estudios de costos, el último de ellos fue realizado en el año 2001, pero los cambios en la práctica clínica, los cambios tecnológicos son demasiados acelerados como para mantener la estructura intacta en 10 años (y en menos también), incluso en el tiempo transcurrido los cambios demográficos y de riesgos sanitarios ocurridos en la población tienen que influir en la intensidad de uso de los factores. El cambio de los precios de los factores, por mucho que se use la inflación, probablemente tampoco se vea reflejado ya que, como se sabe, los precios de los insumos en salud aumentan más rápido que el IPC y, por otro lado, costos de los recursos humanos, que hacen en promedio cerca del $70 \%$ de ellos, están sujetos a las pujas por mejoramientos salariales que se han venido produciendo de cuando en cuando en el sector público de salud.

\section{Conclusiones:}

Los estudios de costos son indispensables en salud, al igual que en cualquier industria, ya que permiten el control y la planificación de las entidades y de sus financiadores. Sus complejidades son mayores que los de la producción de manufacturas por cierto, pero no por ello imposibles. Es preciso afinar siempre las metodologías y declarar los supuestos para tener un juicio sobre la cercanía con la realidad, lo mismo que desarrollar sistemas de información que sigan a los pacientes y sistemas de contabilidad de costos para la valoración de los costos indirectos. Los estudios de costos para las prestaciones básicas deberían ser repetidos al menos cada dos años para tener una apreciación global del manejo financiero de un hospital o un sistema de atención primaria. Además, junto con reconocer los costos reales hace falta adoptar mecanismos elementales para fomentar la eficiencia de la producción de los hospitales, como el modelo de pagos que usa los Grupos Relacionados por el Diagnóstico (GRD) y sus valorizaciones para asignar recursos a los hospitales pagando por desempeño o su equivalente para la atención ambulatoria.

Es sumamente alentador saber que es posible realizar investigación operacional en una situación periférica como es la de un hospital tipo IV, meritorio y 
necesario. Aquí observamos igualmente la energía que otorgan los méritos de los profesionales entrenados en gestión y finanzas en desempeño en los establecimientos de salud.

Evaluar críticamente el impacto de una reforma emblemática, como la de las garantías de 2005 es también una tarea indispensable. Esta reforma justificó un incremento importante en el gasto público en salud con el argumento de la evidencia, la focalización, la priorización y la equidad. La sociedad tiene derecho a saber si esta decisión de política sanitaria fue acertada, si está bien implementada y si sus resultados son favorables. Muchas otras condiciones garantizadas en el Plan de Garantías Explícitas deberían ser analizadas, como ha sido el hecho del Infarto Agudo del Miocardio ${ }^{3}$.

\section{Referencias:}

1. GUTIÉRREZ CG Y MEDINAA. Comparación de los costos reales anuales asociados al manejo ambulatorio de los pacientes hipertensos del programa cardiovascular en el hospital comunitario de Bulnes con el pago de prestaciones de FONASA. Rev Chil Cardiol 2011; 30: 207 - 211
2. http://www.directoriosalud.cl/html/?tag=deuda-hospitalaria

3. NAZZAL N C, CAMPOS T P, CORBALÁN H R, LANAS Z F, BARTOLUCCI J J, SANHUEZA C P, et al, Impacto del plan AUGE en el tratamiento de pacientes con infarto agudo al miocardio con supradesnivel ST, en hospitales chilenos. Rev Méd Chile 2008; 136: 1231-1239 